\title{
List of Appendices
}

2.1 Election campaign information screen 40

2.2 Examples of positive and negative newspaper stories, quotes from campaign, and statements from Campaign Watch Canada 41

2.3 Summary statistics 43

2.4 Multinomial logistic regression results for test of random assignment to treatments 44

3.1 Summary statistics 65

3.2 Multinomial logistic regression results for test of random assignment to treatments 67

3.3 Election instructions 69

3.4 Sample cueing links 69

3.5 Full results for figures 3.2 to $3.7 \quad 70$

4.1 Political-endorsement cueing links 94

4.2 Summary statistics 97

4.3 Multinomial logistic regression results for test of random assignment to treatments 98

4.4 Full results for figures 4.2 to 4.999

5.1 Candidate profiles available to participants 121

5.2 Newspaper story available to participants 123

5.3 Summary statistics 124

5.4 Multinomial logistic regression results for test of random assignment to treatments 125

5.5 Full results for figure $5.2 \quad 126$

6.1 Election campaign information screen 145

6.2 Sample newspaper story and voting record cues 145

6.3 Summary statistics 146

6.4 Multinomial logistic regression results for test of random assignment to treatments 147 
xiv List of Appendices

6.5 Full results for figure $6.1 \quad 148$

6.6 Full multinomial logistic regression results for table 6.2148

7.1 Summary statistics 166

7.2 Multinomial logistic regression results for test of random assignment to treatments 166

7.3 Full results for figure 7.2 167

8.1 Summary statistics 184

8.2 Multinomial logistic regression results for test of random assignment to treatments 185

8.3 Full results for figures 8.1 to $8.4 \quad 186$

8.4 Full results for figures 8.5 to 8.8186

8.5 Full results for figure $8.9 \quad 187$

9.1 Wording of questions about infrastructure-spending-experiment control and treatments 199

9.2 Summary statistics 202

9.3 Multinomial logistic regression results for test of random assignment to treatments 204

9.4 Full results for figures 9.1 to 9.3206

10.1 Summary statistics 220

10.2 Multinomial logistic regression results for test of random assignment to treatments 221

10.3 Full results for figures 10.1 and 10.2222

10.4 Full results for figure 10.3222 